\title{
Fenotipos clínicos en enfermedad pulmonar obstructiva crónica: ¿volver al futuro?
}

\author{
RAFAEL SILVA O.
}

Servicio de Medicina-

Unidad de Enfermedades

Respiratorias, Hospital Regional de Talca.

Facultad de Ciencias de

la Salud, Universidad

Autónoma de Chile.

Recibido el 8 de septiembre de 2011, aceptado el 22 de diciembre de 2011

Correspondencia a: Dr. Rafael Silva O.

Calle 2 Norte 360 Talca. Fono 71-412716 Fax 71- 209306

E-mail: rafaelsilvao@gmail. com

\section{Clinical phenotypes in chronic obstructive pulmonary disease}

Chronic obstructive pulmonary disease (COPD) is a complex and heterogeneous disease, that is defined by the degree of obstruction rendered by the forced expiratory volume in the first second $\left(\mathrm{FEV}_{1}\right)$. This is a good parameter to define the severity of the disease but does not account for its heterogeneity and does not provide a good comprehension of its different clinical behaviors and responses to treatment. Therefore, the classification of these patients in different clinical phenotypes has been attempted, trying to search for common clinical behaviors and responses to treatment. These phenoptypes must be validated in longitudinal studies. Some of the phenotypes detected are COPD with chronic respiratory failure and responsive to home oxygen therapy, COPD with upper lobe emphysema and responsive to volume reduction surgery, COPD with frequent exacerbation behavior, COPD resembling bronchitis and responsive to Roflumilast and possibly, COPD with systemic involvement. Historically, the phenotypes pink puffer, blue bloater, chronic bronchitis were defined. In the next years, we will know if the definitions of these phenotypes will aid in the management of patients with COPD.

(Rev Med Chile 2012; 140: 926-933).

Key words: Bronchitis, chronic; Phenotype; Pulmonary disease, chronic obstructive.
L

a enfermedad pulmonar obstructiva crónica (EPOC) es una enfermedad emergente, progresiva y compleja, tal como se señala en reciente publicación de esta revista ${ }^{1}$.

El diagnóstico, evaluación y manejo de los pacientes con EPOC constituye un dilema importante, al ser considerada esta enfermedad como una limitación crónica del flujo aéreo no completamente reversible, siendo su manejo guiado por la severidad de esta limitación ${ }^{2}$ y, por otra parte, reconociendo la EPOC como un síndrome complejo con numerosos componentes pulmonares $y$ extra pulmonares ${ }^{3}$.

La EPOC es categorizada, desde el punto de vista gravedad, por un parámetro funcional que mide el volumen espiratorio forzado del primer segundo $\left(\mathrm{VEF}_{1}\right)^{2}$, el cual no logra capturar la com- plejidad ni la heterogeneidad de la enfermedad. Se ha intentado caracterizar mejor a estos pacientes, a través de distintos fenotipos ${ }^{4}$, para entender mejor la historia natural, los distintos comportamientos clínicos y las respuestas a las diversas acciones terapéuticas utilizadas.

La identificación y la agrupación de elementos claves de este verdadero "Síndrome EPOC" en sub-grupos clínicamente significativos (fenotipos clínicos) nos puede permitir entender el dilema recién descrito ${ }^{5}$, y constituye el objetivo principal de esta revisión.

\section{Definición}

Un fenotipo es cualquier característica observable de un organismo ${ }^{6}$, sin embargo, estas 
características, por sí mismas, no explican la historia natural o la respuesta terapéutica frente a una enfermedad. Por lo anterior se ha definido ${ }^{5}$ lo que llamaremos fenotipos clínicos en EPOC: "una característica o conjunto de características de la enfermedad que es/son capaces de diferenciar individuos con EPOC y que tienen relación con consecuencias (outcomes) clínicas relevantes, como síntomas, exacerbaciones, respuesta al tratamiento, progresión de la enfermedad o muerte". Por lo tanto, cualquier fenotipo clínico en EPOC debe tener valor predictivo y debe ser validado de manera prospectiva para cada una de las consecuencias clínicas con las que se ha relacionado y, por lo tanto, ser capaz de clasificar a los pacientes en distintos grupos, cada uno con sus respectivos pronósticos y eventuales terapias específicas ${ }^{7}$. Por esto, la validación de los fenotipos clínicos debe ser realizada a través de análisis longitudinales, sin embargo, en la mayoría de las consecuencias clínicas relevantes para esta enfermedad, sólo se han realizado análisis transversales ${ }^{8}$.

Es importante considerar que por las características que presenta la EPOC se podrían considerar como fenotipos algunas consecuencias clínicas, como por ejemplo la gravedad, que en sí misma no es una característica fenotípica, aun cuando una presentación más severa de la EPOC si puede ser consecuencia de un fenotipo determinado ${ }^{5}$.

\section{Antecedentes históricos}

Los distintos fenotipos o sub-tipos de EPOC se han analizado por largos años. El año 1961 aparece la "hipótesis holandesa"9 que afirmaba que en fumadores la hiperreactividad de la vía aérea era el factor clave de la obstrucción bronquial. En 1965 emerge la "hipótesis británica" donde la tos y la expectoración, como marcador de infección bronquial recurrente, era el factor clave de la obstrucción crónica ${ }^{10}$ y Fletcher y Peto, en su clásico artículo publicado en $1977^{11}$, siguen a 792 hombres trabajadores de correos y logran describir la historia natural de la EPOC, detectando aquellos fumadores "susceptibles" de desarrollar EPOC.

El año 2002, Anthonisen publica un seguimiento por 11 años de 5.887 mil sujetos participantes del Lung Health Study, demostrando en los sujetos fumadores una significativa disminución del $\mathrm{VEF}_{1}$, respecto los que dejaron de fumar ${ }^{12}$ y el año 2009
Kohansal $^{13}$ recrea la curva de función pulmonar de Fletcher y Peto, usando datos de la cohorte Framingham Offspring ${ }^{14}$, confirmando que el fumar en forma continua deteriora el $\mathrm{VEF}_{1}$ y causa obstrucción de la vía aérea sólo en un porcentaje de fumadores $y$, al contrario de lo observado por Fletcher y Peto, el observar síntomas respiratorios al inicio del estudio o la aparición de dichos síntomas durante el seguimiento, parecen identificar un fenotipo particularmente susceptible, que desarrolla la enfermedad frente a la noxa tabáquica.

En 1955, Dornhorst ${ }^{15}$ describe los dos fenotipos clásicos de esta enfermedad (Figura 1): "Abotagado Azul” (Blue Bloaters) cuyas características son cianosis, obnubilación, hematocrito superior al $60 \%$, edemas e insuficiencia cardiaca derecha y el "Soplador Rosado" (Pink Puffer) caracterizado por presentar enfisema, pérdida de peso, disnea progresiva y un hematocrito menor de 55\%.

El Simposio CIBA en 1959 describió que en EPOC había una superposición entre la bronquitis crónica, el enfisema y los sub-tipos de asma que se asocian a limitación crónica del flujo aéreo ${ }^{16}$ y en 1985 Burrows ${ }^{17}$ propuso diferenciar los distintos fenotipos de obstrucción crónica del flujo aéreo, tomando como base el pronóstico y define 2 fenotipos: a) el enfisematoso: fumadores no alérgicos ni asmáticos, pero con mala función pulmonar y mayor mortalidad y b) la bronquitis asmática: pacientes asmáticos no fumadores y alérgicos. Sin embargo, esta clasificación no resuelve la sobreposición Asma-EPOC, la que fue primero representada por Snider ${ }^{18}$ en un diagrama no proporcional de Venn, el cual es validado por la American Thoracic Society (ATS) en $1995^{19}$, reafirmando el modelo de obstrucción de vía aérea en la definición de EPOC.

La definición actual, descrita por la Global Initiative for Chronic Obstructive Lung Disease (GOLD) dice que EPOC es "una enfermedad prevenible y tratable, con afectación sistémica extra pulmonar que puede contribuir a la gravedad en algunos pacientes y que el componente pulmonar se caracteriza por una limitación al flujo de aire el cual no es completamente reversible y la limitación al flujo de aire es por lo general progresiva y se asocia con una respuesta inflamatoria pulmonar anómala a partículas o gases nocivos" ${ }^{20}$.

Sin embargo, comienzan a aparecer estudios, como el de Marsh ${ }^{21}$ que describe una clasificación proporcional de los distintos fenotipos clínicos de la EPOC (Figura 2), basados en criterios objetivos, 


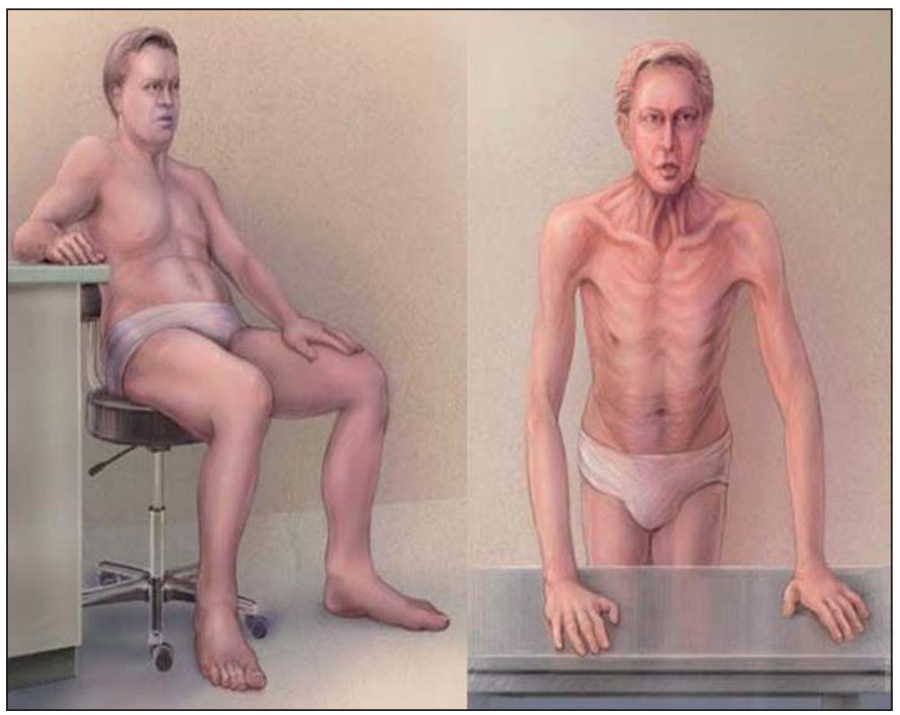

Figura 1. Fenotipos "clásicos" de EPOC: Abotagado azul ("Blue Bloater") y Soplador rosado ("Pink Puffer").

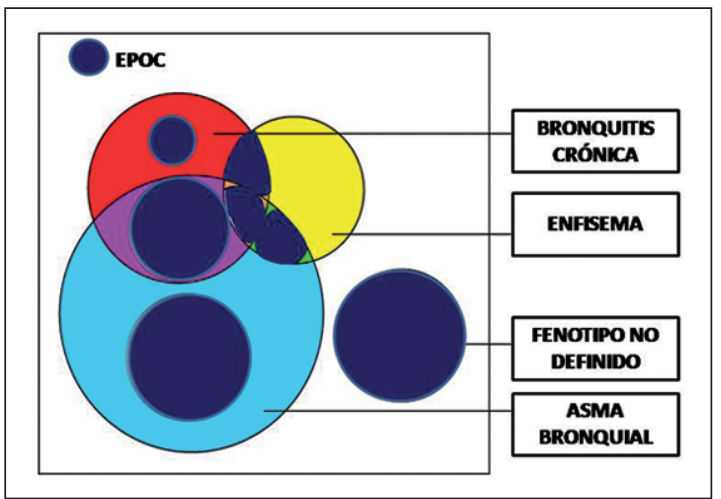

Figura 2. Diagrama proporcional de Venn. Expresiones fenotípicas de Enfermedad Pulmonar obstructiva crónica.

encontrando en su serie que el asma bronquial, con un componente parcialmente reversible, era el fenotipo EPOC dominante que estaba presente en la mitad de los pacientes con $\mathrm{VEF}_{1} / \mathrm{CVF}<0,70$ y que los fenotipos clásicos como el enfisema y la bronquitis crónica, sin asma bronquial, se presentaban en 1 por cada 5 pacientes con $\mathrm{VEF}_{1} /$ $\mathrm{CVF}<0,70$.

Por lo anterior, aparecen más elementos que sugieren, y obligan, a buscar características fenotípicas que nos permitan identificar a grupos de pacientes para, según esas características, poder utilizar terapias más específicas. Esta mirada al pasado, sin duda, nos puede proyectar el manejo futuro de la EPOC.

\section{Heterogeneidad de EPOC}

La EPOC es una enfermedad multicomponente y compleja $a^{22}$ y el $V_{E F}$ no captura ni comunica su heterogeneidad, tal como lo comenta un editorial de New England Journal of Medicine ${ }^{23}$. Esto fue demostrado en el estudio ECLIPSE ${ }^{24}$, estudio de 3 años, longitudinal y observacional, concluyendo que las manifestaciones clínicas de la EPOC son altamente variables y que el grado de obstrucción bronquial, medido a través del $\mathrm{VEF}_{1}$, no logra expresar la complejidad de la enfermedad. Aun más, algunas manifestaciones extrapulmonares asociadas a EPOC, tal como la pérdida de peso y la disfunción de la musculatura esquelética, influyen el curso de la enfermedad, independiente del $\mathrm{VEF}_{1}^{25} \mathrm{y}$ el tratamiento de estas condiciones, por ejemplo a través de rehabilitación, contribuye a mejorar el pronóstico y el bienestar de estos pacientes, sin mejora concomitante de la función pulmonar ${ }^{26}$.

Además el estudio ECLIPSE demostró que en cada estadio de gravedad GOLD hay una alta variabilidad de los síntomas, de la tolerancia al ejercicio y de la frecuencia de las exacerbaciones, de tal forma que en cada estadio hay pacientes con mucha o poca afectación clínica, por lo que es posible que lo que hoy denominamos EPOC, sea en la realidad muchas "pequeñas EPOC", cada una con diferente historia natural y respuesta terapéutica ${ }^{27}$. 
Fenotipos clínicos de EPOC - R. Silva

\section{Características fenotípicas de la EPOC}

No cabe duda que el sólo utilizar un parámetro funcional $\left(\mathrm{VEF}_{1} / \mathrm{CVF}<0,70\right)$, para el diagnóstico de EPOC, abre la puerta a clasificar como tal a un gran número de pacientes diversos, con patrones clínicos distintos. Obviamente hemos sobresimplificado el diagnóstico, con una finalidad: poder disminuir el sub-diagnóstico y mejorar la detección precoz, especialmente a nivel de atención primaria $^{28}$. Esta sobresimplificación nos ha hecho pasar desde el "abotagado azul", el "soplador rosado", la bronquiolitis tabáquica, la bronquitis asmatiforme, las bronquiectasias en fumadores, etc. al concepto unitario de EPOC como $\mathrm{VEF}_{1} /$ $\mathrm{CVF}<0,70$ post-broncodilatador, adoptando un patrón de tratamiento igual para todos, basados en la severidad de la obstrucción. Por esta razón algunos autores sugieren pasar de una indicación de tratamiento por gravedad, como lo hacemos hoy, a una indicación de tratamiento por fenotipo, como pudiera ser en el futuro ${ }^{29}$.

Pero no son muchos los fenotipos clínicos de EPOC que cumplen con las características incluidas en la definición descrita y que, por lo tanto, tienen validación clínica comprobada. Estos fenotipos son los siguientes:

El primer fenotipo, y el más antiguo descrito, que cumple con la característica de tener valor predictivo y validarse de manera prospectiva para una consecuencia clínica específica, lo constituyen los pacientes con EPOC e insuficiencia respiratoria crónica $^{30}$, en los cuales el uso de oxígeno domiciliario, por lo menos durante 15 horas diarias, ha mostrado efecto positivo sobre la mortalidad a los 60 meses $^{31,32}$, mientras que esta misma acción no tiene ningún efecto beneficioso sobre el pronóstico, si se usa en pacientes sin la indicación precisa $^{33,34}$.

Un segundo fenotipo, más recientemente descrito, que cumple dichos requisitos, es la respuesta positiva a la cirugía de reducción de volumen, la cual ha mostrado su eficacia en un subgrupo concreto de pacientes portadores de EPOC, específicamente en aquellos portadores de enfisema localizado en lóbulos superiores y con mala tolerancia al ejercicio después de un programa de rehabilitación ${ }^{35}$.

Un tercer fenotipo con la característica señalada, es un grupo de pacientes con EPOC moderada y severa $\left(\mathrm{VEF}_{1}<50 \%\right)$ con tos crónica y produc- ción permanente de esputo, es decir, con características de bronquitis crónica, que con el uso de roflumilast, un inhibidor de la fosfodiesterasa-4, mejoran su función pulmonar y disminuyen el número de exacerbaciones anuales ${ }^{36,37}$.

Estos ejemplos son claramente insuficientes para entender el comportamiento complejo de los pacientes portadores de EPOC y por esa razón se han desarrollado protocolos para identificar mejor estos eventuales fenotipos.

Uno de estos protocolos ha sido desarrollado por Hurst et al..$^{38}$ que utilizó la cohorte del estudio ECLIPSE para aclarar si aquellos pacientes con frecuentes exacerbaciones corresponden a un fenotipo clínico con comportamiento estable e independiente de la severidad de la obstrucción de la vía aérea. Se analizó la frecuencia de exacerbaciones de EPOC y su asociación con diversos componentes de la enfermedad en un seguimiento por 3 años a 2.138 pacientes. Este estudio definió como exacerbación de EPOC a un "evento que requiere el uso de antibióticos y/o corticoides sistémicos o que requiere hospitalización (exacerbación EPOC severa)" y como "exacerbador frecuente" a aquellos pacientes con más de 2 exacerbaciones/año. Se encontró que en el primer año de seguimiento los episodios de exacerbación eran más frecuentes y severos, a mayor grado de obstrucción bronquial. El fenotipo "exacerbador frecuente" se mantuvo constante durante los 3 años de seguimiento y se encontró que la frecuencia de exacerbaciones del año anterior era el mejor predictor de la frecuencia de las exacerbaciones futuras, concluyendo los autores que este fenotipo exacerbador frecuente constituye un fenotipo clínico especifico que puede ser fácilmente identificable por la anamnesis. Hay, sin embargo, algunas interrogantes respecto este punto ${ }^{39}$ ¿hay en la historia natural de estos enfermos paso del estado exacerbador frecuente al estado no exacerbador (ninguna exacerbación al año)? Este eventual paso de un estado a otro, ¿se puede intervenir terapéuticamente? Futuros estudios posiblemente nos darán las respuestas.

Otro estudio realizado con el objetivo de identificar y validar fenotipos clínicos en EPOC es el publicado por García-Aymerich a principios del año $2011^{40}$, en el cual reclutaron 322 pacientes hospitalizados por exacerbación EPOC y los estudiaron 3 meses post-alta, en condiciones de estabilidad clínica. Evaluaron nivel de síntomas 
(tos y esputo), calidad de vida (cuestionario de San George) y diversas variables de función pulmonar (volúmenes pulmonares, capacidad de difusión, test de marcha de $6 \mathrm{~min}$, disnea), capacidad de ejercicio (consumo de oxígeno por test cardiopulmonar), estado nutricional, imagen torácica por tomografía computada, varios marcadores inflamatorios en sangre periférica (proteína $\mathrm{C}$ reactiva, fibrinógeno, factor de necrosis tumoral, interleukina 6) y en secreciones bronquiales (interleukina 8 , recuento de neutrófilos) y función cardiaca por eco cardiografía. Utilizando la técnica estadística cluster, se determinaron grupos con características comunes, los que se relacionaron a 2 consecuencias clínicamente relevantes, como la mortalidad y las hospitalizaciones. Identificaron y validaron 3 fenotipos específicos: a) EPOC con afectación respiratoria grave; b) EPOC con afectación respiratoria moderada; c) EPOC sistémico, con alta prevalencia de obesidad, alteración cardiovascular, diabetes e inflamación sistémica. Obviamente estos resultados no se pueden proyectar a toda la población de pacientes con EPOC, sino que sólo a aquellos con primera hospitalización por exacerbación. Estos resultados son un avance, particularmente al identificar y validar el "fenotipo sistémico".

Hay varios estudios que describen distintos fenotipos de EPOC que no cumplen con el requisito de tener valor predictivo y validarse de manera prospectiva con una consecuencia clínica específica. Algunos de estos son los siguientes:

Un reporte inicial del estudio ECLIPSE ${ }^{41}$ muestra que los pacientes con exacerbaciones severas de EPOC tienen enfermedad más avanzada, mayor alteración de calidad de vida y sufren más depresión. Otro reporte de esta cohorte ${ }^{42}$ muestra que pacientes que caminan menos de 350 metros en el test de marcha de 6 min tienen más enfisema en la tomografía computada de tórax, más depresión y mayor disnea.

Un estudio realizado por un grupo japonés ${ }^{43}$ examinó las tomografías computadas de tórax de 85 pacientes EPOC estables, evaluando cambios enfisematosos y grosor de la pared bronquial. Clasificó a los pacientes en 1) fenotipo A: ausencia de enfisema o poco enfisema con o sin engrosamiento bronquial; 2) fenotipo E: enfisema sin engrosamiento bronquial; 3) fenotipo M: enfisema con engrosamiento bronquial. El fenotipo A tiene una alta prevalencia de pacientes no fumadores, sibilancias, aumento del índice de masa corporal y reversibilidad con $\beta 2$-agonistas inhalados, comparado con los otros 2 fenotipos. Además detectaron que el engrosamiento bronquial en el fenotipo A y M se asocia significativamente a una respuesta positiva a los corticoides inhalados (con reversibilidad a largo plazo) y al hallazgo de eosinofília en esputo.

Finalmente, Burger ${ }^{44}$, reclutó 322 pacientes con EPOC, utilizando también la metodología cluster, con el uso de análisis de componentes principales. Analizó 8 variables: edad, grado de consumo de tabaco, $\mathrm{VEF}_{1}$ (\% pre-dicho), índice de masa corporal, exacerbaciones, disnea (MMRC), estado de salud (cuestionario de Saint George) y síntomas depresivos. Describieron 4 fenotipos: 1) Joven/Severo; 2) Viejo/Leve; 3) Joven/Moderado; 4) Viejo/ Severo. Una de las principales conclusiones de este estudio fue que pacientes con similar obstrucción de vía aérea $\left(\mathrm{VEF}_{1}\right)$, tienen diferente nivel de síntomas (disnea) y consecuencias (número de exacerbaciones y mortalidad) y que el fenotipo 4 tiene una mayor prevalencia de insuficiencia cardiaca y sobrepeso.

Sin embargo, hay algunas opiniones distintas respecto mirar la EPOC en función de fenotipos clínicos. Rodríguez-Roisin ${ }^{45}$ afirma que por más de 25 años la comunidad médica pivotó sobre dos fenotipos universales de EPOC, los ya descritos tipo A (enfisema pulmonar) y tipo B (bronquitis crónica). Hoy esta tipificación está obsoleta, según este autor, por su baja utilidad clínica, afirmando que la severidad de la obstrucción bronquial, la disnea y los antecedentes de exacerbaciones en los pacientes con EPOC deben seguir siendo los tres pilares fundamentales de la estrategia terapéutica, más que buscar estrategias frente a fenotipos determinados.

\section{El futuro y la caracterización fenotípica}

Durante los próximos años posiblemente aumente la identificación y validación de muchos fenotipos clínicos de EPOC. Este proceso requiere identificar previamente su relevancia respecto a una consecuencia clínica, con elementos clínicos, fisiológicos e imagenológicos similares que den lugar a una caracterización molecular o biológica, para desarrollar terapias específicas para una población determinada de pacientes con EPOC 5 . Esto 
puede ser tan ideal que al final podríamos no tener una terapia específica para cada fenotipo. Por otro lado, un fenotipo puede no ser nítido en etapas precoces de la enfermedad o bien cada individuo podría desarrollar más de un fenotipo clínico 5 .

Por otro lado, y tal como se ha comentado en esta revista ${ }^{46}$, se están desarrollando conceptos de ingeniería y física como las "redes libres de escala" y los "sistemas complejos", que junto a los avances alcanzados en ciencias básicas, el desarrollo de herramientas informáticas y de imagenología, se conjugan para desarrollar la Medicina P4 (medicina predictiva, personalizada, preventiva y participatoria) con el fin de romper los paradigmas de la investigación médica actual. Esto se ha proyectado hacia la $\mathrm{EPOC}^{47}$ para permitir una mejor comprensión de las múltiples dimensiones de esta enfermedad. La relación de la EPOC con otras enfermedades es muy relevante y de alto interés en la actualidad y la información que pueda resultar de la Medicina P4 permitirá abordar mejor su complejidad con el fin de integrarlas en la práctica clínica rutinaria de esta enfermedad ${ }^{47}$. En la medida que mejore el conocimiento de las redes celulares y moleculares, es posible que en el futuro algunas comorbilidades de la EPOC pasen a ser parte integral de un fenotipo determinado ${ }^{48}$.

\section{Conclusión}

La EPOC es una enfermedad heterogénea y compleja. La búsqueda de fenotipos nos permitirá caracterizar mejor a estos pacientes para tener terapias más específicas que permitan cambiar su historia natural. Estos fenotipos deben ser validados en forma prospectiva/longitudinal y tener valor predictivo frente a cada consecuencia clínica relacionada.

Hay algunos fenotipos clínicos que cumplen este requisito: EPOC con falla respiratoria y oxígenoterapia domiciliaria, EPOC con enfisema lóbulos superiores y cirugía de reducción de volumen, EPOC tipo bronquitis crónica y uso de roflumilast, EPOC exacerbador frecuente $y$, posiblemente, EPOC con efectos sistémicos.

Es muy posible que con información relevante del pasado y las nuevas formas de integrar el conocimiento actual, como la Medicina P4, esta enfermedad encontrara, sin duda, terapias de alta efectividad en el futuro.

\section{Referencias}

1. Silva R. Enfermedad pulmonar obstructiva crónica: Mirada actual a una enfermedad emergente. Rev Med Chile 2010; 138: 1544-52.

2. Rabe K, Hurd S, Anzueto A, Barnes P, Buist S, Calverley $\mathrm{P}$, et al. Global Strategy for the Diagnosis, Management, and Prevention of Chronic Obstructive Pulmonary Disease. Am J Respir Crit Care Med 2007; 176: 532-55.

3. Barnes P, Celli B. Systemic manifestations and comorbidities of COPD. Eur Respir J 2009; 33: 1165-85.

4. Vestbo J, Anderson W, Coxson H, Crim C, Dawber F, Edwards L, et al. Evaluation of COPD Longitudinally to Identify Predictive Surrogate End-points (ECLIPSE). Eur Respir J 2008; 31: 869-73.

5. Han M, Agusti A, Calverley P, Celli B, Criner G, Curtis J, et al. Chronic Obstructive Pulmonary Disease Phenotypes. The Future of COPD. Am J Respir Crit Care Med 2010; 182: 598-604.

6. Freimer N, Sabatti C. The human phenome project. Nat Genet 2003; 34: 15-21.

7. Agusti A, Sobradillo P, Celli B. Addressing the Complexity of Chronic Obstructive Pulmonary Disease. Am J Respir Crit Care Med 2011; 183: 1129-37.

8. Jones P, Agusti A. Outcomes and markers in the assessment of chronic obstructive pulmonary disease. Eur Respir J 2006; 27: 822-32.

9. Orie N, Sluiter H, de Vries K. The host factor in bronchitis. Orie, NGM Sluiter, HJ eds. Bronchitis 1961; 43-59 Royal van Gorcum. Assen, the Netherlands.

10. Reid L. The role of chronic bronchitis in the production of "chronic obstructive pulmonary emphysema". J Am Med Womens Assoc 1965; 20: 633-8.

11. Fletcher C, Peto R. The Natural History of Chronis Bronquitis and Enphysema. BMJ 1977; 1: 1645-8.

12. Anthonisen N, Connett J, Murray R. Smoking and Lung Function of Lung Health Study Participants after 11 Years. Am J Respir Crit Care Med 2002; 166: 675-9.

13. Kohansal R, Martínez-Camblor P, Agusti A, Buist S, Mannino D, Soriano J. The Natural History of Chronic Airflow Obstruction Revisited:An Analysis of the Framingham Offspring Cohort. Am J Respir Crit Care Med 2009; 80: 3-10.

14. Kannel W, Feinleib M, McNamara P, Garrison R, Castelli W. An investigation of coronary heart disease in families. The Framingham offspring study. Am J Epidemiol 1979; 110: 281-90.

15. Dornhorst A. Respiratory Insufficiency. Lancet 1955; 268 (6876): 1185-7.

16. Anon. Terminology, definitions, and classification of chronic pulmonary emphysema and related conditions. 
A report of the conclusions of a Ciba guest symposium. Thorax 1959; 14: 286-99.

17. Burrows B, Bloom J, Traver G, Cline M. The course and prognosis of different forms of chronic airways obstruction in a sample from the general population. N Engl J Med 1987; 317: 1309-14.

18. Snider G. Chronic obstructive pulmonary disease: a definition and implications of structural determinants of airflow obstruction for epidemiology. Am Rev Respir Dis 1989; 140 (3 Pt 2): S3-8.

19. American Thoracic Society. Standards for the diagnosis and care of patients with chronic obstructive pulmonary disease. Am J Respir Crit Care Med 1995; 152 (5 Pt 2): S77-121.

20. Rabe K, Hurd S, Anzueto A, Barnes P, Buist S, Calverley $\mathrm{P}$, et al. Global Strategy for the Diagnosis, Management, and Prevention of Chronic Obstructive Pulmonary Disease. Am J Respir Crit Care Med 2007; 176: 532-55.

21. Marsh S, Travers J, Weatherall M, Williams M, Aldington S, Shirtcliffe P, et al. Proportional classifications of COPD phenotypes. Thorax 2008; 63: 761-7.

22. Agusti A. COPD, a multicomponent disease: implications for management. Respir Med 2005; 99: 670-82.

23. Reilly J. COPD and Declining FEV1-Time to Divide and Conquer? N Engl J Med 2008; 359: 1616-8.

24. Agusti A, Calverley P, Celli B, Coxson H, Edwards L, Lomas D, et al. Characterisation of COPD heterogeneity in the ECLIPSE cohort. Respiratory Research 2010; 11: 122-36.

25. Schols A, Slangen J, Volovics L, Wouters E. Weight loss is a reversible factor in the prognosis of chronic obstructive pulmonary disease. Am J Respir Crit Care Med 1998; 157: 1791-7.

26. Ries A, Kaplan R, Limberg T, Prewitt L. Effects of pulmonary rehabilitation on physiologic and psychosocial outcomes in patients with chronic obstructive pulmonary disease. Ann Intern Med 1995; 122: 823-32.

27. Rennard S, Vestbo J. The Many "Small COPDs": COPD Should Be an Orphan Disease. CHEST 2008; 134: 623-7.

28. Tálamo C, Montes de Oca M, Halbert R, Pérez-Padilla R, Jardim J, Muiño A, et al. Diagnostic Labeling of COPD in Five Latin American Cities. Chest 2007; 131: 60-7.

29. Miravitlles M. Tratamiento individualizado de la EPOC: una propuesta de cambio. Arch Bronconeumol 2009; 45 Suppl 5: 27-34.

30. Nocturnal Oxygen Therapy Trial Group. Continuous or nocturnal oxygen therapy in hypoxemic chronic obstructive lung disease. A clinical trial. Ann Intern Med 1980; 93: 391-8.

31. Report of the Medical Research Council Working Par- ty. Long term domiciliary oxygen therapy in chronic hypoxic cor pulmonale complicating chronic bronchitis and emphysema. Lancet 1981; i: 681-5.

32. Nocturnal Oxygen Therapy Trial Group. Continuous or nocturnal oxygen therapy in hypoxemic chronic obstructive lung disease. A clinical trial. Ann Intern Med 1980; 93: 391-8.

33. Górecka D, Gorzelak K, Sliwinski P, Tobiasz M, Zielinski J. Effect of long term oxygen therapy on survival in patients with chronic obstructive pulmonary disease with moderate hypoxaemia. Thorax 1997; 52: 674-9.

34. Zielinski J. Long-term oxygen therapy in COPD patients with moderate hypoxaemia: does it add years to life? Eur Respir J 1998; 12: 756-8.

35. National Emphysema Treatment Trial Research Group. A randomized trial comparing lung-volume-reduction surgery with medical therapy for severe emphysema. $\mathrm{N}$ Engl J Med 2003; 348: 2059-73.

36. Fabbri L, Calverley P, Izquierdo-Alonso J, Bundschuh D, Brose M, Martínez F, et al. Roflumilast in moderateto-severe chronic obstructive pulmonary disease treated with longacting bronchodilators: two randomised clinical trials. Lancet 2009; 374: 695-703.

37. Calverley P, Rabe K, Goehring U, Kristiansen S, Fabbri L, Martínez F. Roflumilast in symptomatic chronic obstructive pulmonary disease: two randomised clinical trials. Lancet 2009; 374: 685-94.

38. Hurst J, Vestbo J, Anzueto A, Locantore N, Ullerova $\mathrm{H}$, Tal-Singer R, et al. Susceptibility to exacerbation in chronic obstructive pulmonary disease. $\mathrm{N}$ Engl J Med 2010; 363: 1128-3.

39. Sobradillo P, García-Aymerich J, Agustí A. Fenotipos Clínicos de la EPOC. Arch Bronconeumol 2010; 46 (Supl 11): 8-11.

40. García-Aymerich J, Gómez F, Benet M, Farrero E, Basagaña X, Gayete A, et al. Identification and prospective validation of clinically relevant chronic obstructive pulmonary disease (COPD) subtypes. Thorax 2011; 66: 430-7.

41. Anzueto A, Müllerova H, Locantore N, Hurst J, Wedzicha J, Vestbo J. Occurrence And Descriptors Of Severe COPD Exacerbations In The ECLIPSE Cohort. Am J Respir Crit Care Med 181; 2010: A2388.

42. Spruit M, Watkins M, Edwards L, Vestbo J, Calverley P, Pinto-Plata V, et al. Determinants of poor 6-min walking distance in patients with COPD: The ECLIPSE cohort. Respiratory Medicine 2010; 104: 849-57.

43. Kitaguchi Y, Fujimoto K, Kubo K, Honda T. Characteristics of COPD phenotypes classified according to the findings of HRCT. Respir Med 2006; 100: 1742-52.

44. Burgel P, Paillasseur J, Caillaud D, Tillie-Leblond I, 
Chanez P, Escamilla R, et al. Clinical COPD phenotypes: a novel approach using principal component and cluster analyses. Eur Respir J 2010; 36: 531-9.

45. Rodríguez-Roisin R. Glucocorticoides inhalados en la EPOC por fenotipo en lugar de gravedad. Argumentos en contra. Arch Bronconeumol 2011; 47 (6): 269-70.

46. Silva R. Investigación médica, mineros chilenos y "Medicina P4". Rev Med Chile 2011; 139: 550-1.
47. Agustí A, Sobradillo P, Celli B. Addressing the Complexity of Chronic Obstructive Pulmonary Disease From Phenotypes and Biomarkers to Scale-Free Networks, Systems Biology, and P4 Medicine. Am J Respir Crit Care Med 2011; 183: 1129-37.

48. Park J, Lee D, Christakis N, Barabasi A. The impact of cellular networks on disease comorbidity. Mol Syst Biol 2009; 5: 262. 